\title{
Intraoperative IGS/CT Updates for Complex Endoscopic Frontal Sinus Surgery
}

\author{
Sri Kiran Chennupati Bradford A. Woodworth James N. Palmer \\ Noam A. Cohen David W. Kennedy Alexander G. Chiu \\ Division of Rhinology, Department of Otorhinolaryngology, Head and Neck Surgery, University of Pennsylvania, \\ Philadelphia, Pa., USA
}

\section{Key Words}

Image guidance $\cdot$ Intraoperative CT $\cdot$ Endoscopic sinus

surgery $\cdot$ Computer-aided surgery

\begin{abstract}
Introduction: Computer-aided surgery (CAS) systems integrate endoscopic visualization with real-time localization based on preoperative imaging. One short-coming of the current systems is the lack of real-time, radiographic assessment of surgical changes. We describe the potential applications of $x \mathrm{XAT}^{\mathrm{TM}}$, a new intraoperative mobile volume $\mathrm{CT}$ scanner and its utility in providing surgical navigation updates in the operating room. Methods: A case report is presented describing how intraoperative $C T$ updates were used in a complicated, revision endoscopic frontal sinus surgery. Results: A 53-year-old male with Samter's triad and a history of multiple previous endoscopic sinus surgeries presented with recurrent, symptomatic nasal polyposis and right-sided frontal headaches. Preoperative CT scans showed a large type III frontal recess cell obstructing the frontal sinus. He underwent revision endoscopic surgery with the use of CAS and the $x$ CAT intraoperative CT scanner. Use of the intraoperative CT scanner to update preoperative images was extremely helpful in the endoscopic dissection of the frontal recess cell and in assessing completeness of dissection along the anterior skull base. Conclusion: The xCAT intraoperative
\end{abstract}

\section{KARGER}

Fax +41613061234 E-Mail karger@karger.ch www.karger.com
(ㄷ) 2008 S. Karger AG, Basel

0301-1569/08/0704-0268\$24.50/0

Accessible online at:

www.karger.com/orl mobile volume CT scanner shows promise in providing realtime updates to surgical navigation. This may prove to be a valuable tool in endoscopic sinus surgery, especially in complex revision and frontal sinus cases.

Copyright $\odot 2008$ S. Karger AG, Basel

\section{Introduction}

With the advent of surgical navigation in the late 1980s, endoscopic surgeons have increasingly utilized this technology for intraoperative localization and preoperative planning for frontal recess dissections. Finecut CT scans, often $1 \mathrm{~mm}$ in section, allow for greater understanding of anatomy that has been distorted by previous surgery, polypoid mucosa and/or anatomical variants. One of the drawbacks of this system is that surgical navigation is based on a CT scan obtained prior to the operation. Real-time updates to confirm exposure of the true frontal recess and removal of obstructing cells have until now been impractical to obtain.

The xCAT ${ }^{\mathrm{TM}}$ (Xoran Technologies, Ann Arbor, Mich., USA) is a compact, portable volume CT scanner that can provide $0.4-\mathrm{mm}$-thick images of the paranasal sinuses in less than $3 \mathrm{~min}$. Coupled with existing image guidance systems, the xCAT provides real-time surgical updates that can prove useful in difficult bony dissections. The 
Fig. 1. Mobile volume CT scanner in use in the operating room.

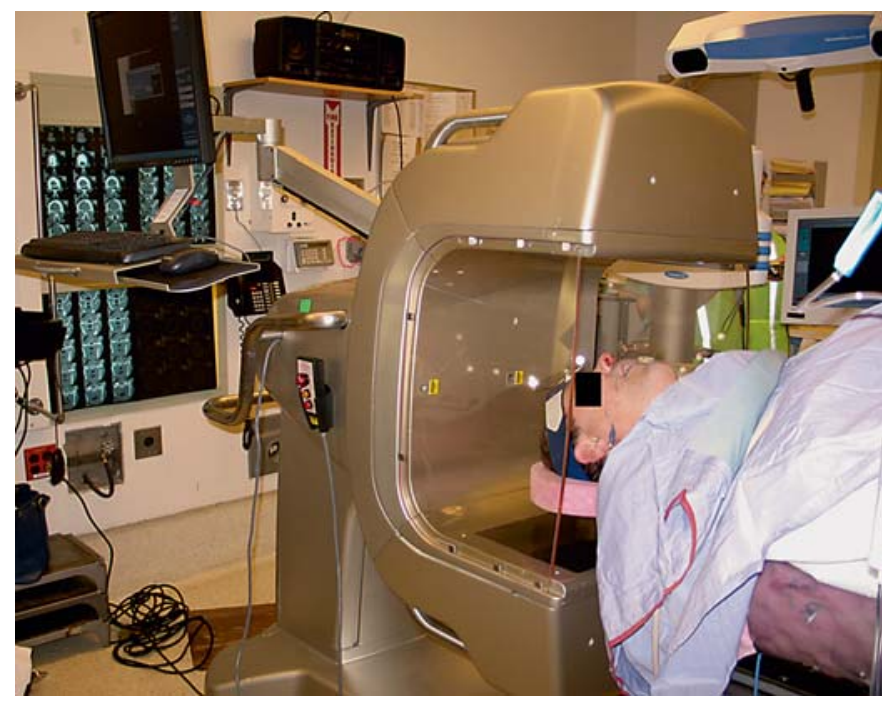

Fig. 2. A screen shot from the BrainLAB surgical navigational system. The row of CT scans on the left is from the intraoperative CT scanner. The row of CT scans on the right represents the preoperative CT scan. The black arrows point to the vertical bony wall separating the right type III frontal recess cell from the right frontal sinus. Note the intraoperative scan on the left shows partial removal of that bony partition.
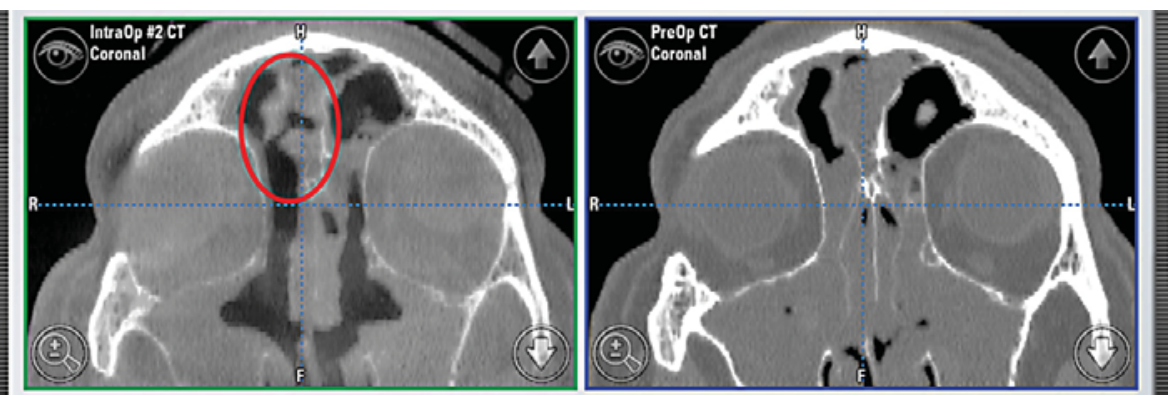

following illustrates a case in which this technology aided in the dissection of a large, obstructing frontal recess cell that was initially mistaken for the frontal sinus.

\section{Case Example}

A 53-year-old male with Samter's triad and a history of two previous endoscopic sinus surgeries presented with nasal obstruction, anosmia and bilateral frontal pressure headaches. Preoperative CT scans showed a large type III frontal recess cell obstructing the right frontal sinus and residual bony partitions along the anterior skull base. He underwent revision endoscopic surgery with the use of a BrainLAB (BrainLAB, Heimstetten, Germany) computer-aided system and the xCAT intraoperative CT scanner (fig. 1).

Endoscopic dissection was carefully performed along the skull base to first address the remaining ethmoid bony partitions. Using a $70^{\circ}$ endoscope and frontal sinus curette and probes, the supposed right frontal recess was cleared. To confirm the correct identification of the frontal sinus, a radiopaque cottonoid was placed in this cavity and an intraoperative CT scan was performed. The DICOM data was downloaded and transferred to the BrainLAB system. Next, the information was transformed to a volumetric data set and fused with the existing data set from the preoperative CT scan. The updated images showed the radiopaque marker to be within the type III frontal cell, with the true entrance to the frontal sinus medial and blocked by a vertical bony partition.

Further dissection of the frontal recess then ensued. The medial vertical bony partition was identified and removed using the updated image-guided scans. The true frontal sinus was identified and the bony wall between the sinus and the type 3 frontal recess cell was removed as far superiorly as the instruments allowed. A second intraoperative scan was performed with a radiopaque marker placed within the supposed frontal sinus. This time, after fusion of the new data set with the BrainLAB system, the radiopaque marker was confirmed to be within the true frontal sinus (fig. 2).

\section{Discussion}

Intraoperative imaging acquisition with computer assistance is not a novel concept. As early as 1980, intraoperative CT imaging was performed using a CT-dependent frame that served as fiducial markers for the computer 
during neurosurgical procedures [1]. Because of the impractical nature of using large CT scanners in the operating room and the resulting large doses of radiation patients were receiving, intraoperative imaging was abandoned for one preoperative imaging sequence that could be applied to stereotactic navigational systems.

The advent of new technology has now made intraoperative CT scanning with real-time surgical navigation updates possible and practical. A compact, portable CT scanner called the xCAT provides real-time intraoperative CT scans of the sinuses and skull base. The system consists of a cone-beam CT scanner that contains an Xray source and detector mounted on a rotating scanning arm, a personal computer with an integrated widescreen monitor, and an image-processing unit. During one rotation of the scanning arm, the detector collects the flux of $\mathrm{X}$-rays that have passed through the patient. Cone-beam CT scanners utilize a two-dimensional multi-row detector, which allows for a single rotation of the gantry to generate a scan of the entire head, as compared to conventional, full-body 'fan-beam' CT scanners whose multiple 'slices' must be stacked to obtain a complete image. Thus, this system displays scans on its integrated monitor in less than $3 \mathrm{~min}$.

Cone-beam technology utilizes X-rays much more efficiently, requires far less electrical energy, and allows for the use of more compact X-ray components than fanbeam technology [1]. This system creates CT images with isotropic spatial resolution of $0.4 \mathrm{~mm}$, and its customized field of view is optimized for scanning the sinuses and skull base. The effective radiation dose to the patient is as low as 0.25 millisievert (mSV), while the radiation dose from a full-body CT scanner is on average 10 times higher [2].

Revision frontal sinus surgery remains a significant challenge to the endoscopic surgeon. One of the main reasons for its difficulty lies in the complexity and often variable anatomy surrounding the frontal recess. Originally described by Owen and Kuhn [3], frontal recess cells are ethmoid cells that lie above the agger nasi and pneumatize into the frontal sinus. A type III frontal recess cell is a single large cell that pneumatizes well into the true frontal sinus. These are typically the most difficult to dissect out endoscopically and often a frontal sinus trephine is needed to fully remove the cell. When performing an endoscopic dissection, the top of these cells can bear a strong resemblance to and easily be confused with the frontal sinus. Illumination of the frontal sinus and confirmation with image guidance machines using preoperative data are imperfect methods to ensure proper dissection of these cells. As illustrated in the above case, the use of a mobile, intraoperative CT scanner, coupled with surgical navigation, is the ideal way to confirm the thoroughness of a difficult frontal recess dissection.

\section{Conclusion}

The xCAT intraoperative mobile volume CT scanner can provide real-time updates to surgical navigation. This may prove to be a valuable tool in complex revision and frontal sinus cases. Future studies should focus on additional applications and situations where this new technology will provide for safer and more complete surgeries.

References

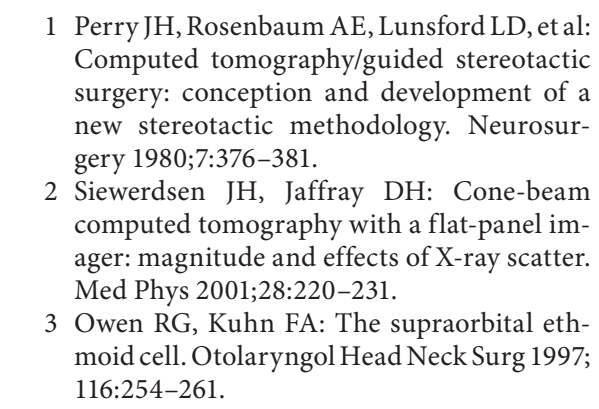

Chennupati/Woodworth/Palmer/Cohen/ Kennedy/Chiu 